\title{
When is definitive radiotherapy the preferred treatment for head and neck squamous cell carcinoma?
}

\author{
William M. Mendenhall ${ }^{1}$ Primož Strojan ${ }^{2}$ Avraham Eisbruch ${ }^{3}$. \\ Robert Smee $^{4} \cdot$ Alessandra Rinaldo $^{5} \cdot$ Alfio Ferlito $^{6}$
}

Received: 27 April 2015/Accepted: 19 May 2015/Published online: 30 May 2015

(C) Springer-Verlag Berlin Heidelberg 2015

\section{Introduction}

Head and neck mucosal squamous cell carcinoma (SCC) may be treated with surgery and/or radiotherapy (RT) with or without adjuvant chemotherapy. The optimal treatment strategy depends not only on the location and locoregional extent of the SCC, but also on anticipated functional results, expertise of treatment teams and logistical considerations. The following is a brief discussion of head and neck mucosal SCCs where definitive RT is the preferred, or at least one of the preferred treatment options. We do not address advanced incompletely resectable SCCs where definitive RT is the treatment of choice by default. In general, concomitant platinum-based chemotherapy is indicated for patients with stage III-IV disease [1]. In the era of improved diagnostic follow-up modalities the use of planned neck dissection is limited only to patients not achieving complete response in the neck at 8-12 weeks after treatment completion as

This article was written by members of the International Head and Neck Scientific Group (www.IHNSG.com).

Alfio Ferlito

a.ferlito@uniud.it

1 Department of Radiation Oncology, University of Florida, Gainesville, FL, USA

2 Department of Radiation Oncology, Institute of Oncology, Ljubljana, Slovenia

3 Department of Radiation Oncology, University of Michigan, Ann Arbor, MI, USA

4 Department of Radiation Oncology, The Prince of Wales Cancer Centre, Sydney, NSW, Australia

5 University of Udine School of Medicine, Udine, Italy

6 Via Firenze 11, Selvazzano Dentro, 35031 Padua, Italy determined on post-RT computed tomography (CT) or positron emission tomography (PET-CT) [2].

\section{Nasopharynx}

Although early-stage nasopharyngeal carcinomas are potentially resectable, the preferred treatment for the vast majority of these patients is RT alone or in combination with chemotherapy. Probably, the most informative data was presented by Lee et al. in their analysis of a large cohort of 1593 consecutive nasopharyngeal cancer patients treated between 1994 and 2010 in Hong Kong [3]. They observed a significant improvement in survival and toxicity when moving from two-dimensional RT to three-dimensional RT and intensity-modulated radiotherapy (IMRT): the resultant 5-year disease-specific survival rates (for all stages together) were 78,81 and $85 \%$, respectively, and neurologic toxicity rates were $7.4,3.5$ and $1.8 \%$, respectively. However, the RT technique alone did not contribute to this improvement, but also advances in imaging and the increasing use of chemotherapy which was, on the other hand, an important cause of hearing impairment. With more potent chemotherapy and/or innovative methods to overcome the plateau in the local control reached with present treatment strategies, and with optimization of RT dose constraints for different organs at risk, further improvement of the therapeutic ratio could be expected [3].

\section{Oropharynx}

Patients with oropharyngeal cancer, particularly those arising in the tonsil and base of tongue, may be treated with RT or transoral resection. So far, no randomized trial compared face-to-face primary surgery [with or without 
adjuvant (chemo)radiation] and primary (chemo)RT. Excellent treatment results in RT-based series [4-6], particularly in the era of IMRT and the high proportion of HPV-positive tumors, makes it a good treatment alternative for the majority of patients with oropharyngeal SCCs or even a preferred treatment option in many institutions around the globe [7-10]. In view of significant treatment morbidity and mortality associated with high-dose chemo$\mathrm{RT}$, as well as in patients treated with open radical surgery with or without tissue reconstruction [11-13], transoral robotic surgery (TORS) was introduced which resulted in high local control rates and favorable survival outcomes [14]. However, even when using this most advanced surgical technique, adjuvant RT is needed in the majority of patients, mainly due to the advanced $\mathrm{N}$-stage of the disease, with concurrent use of platinum-based chemotherapy in those with positive surgical margins and/or extracapsular tumor spread. Thus, when adjuvant treatment is added to TORS, the overall treatment toxicity is increased. Recently, de Almeida et al. compared the effectiveness and adverse effects of IMRT ( 8 series with 1287 patients, $43 \%$ received chemotherapy and $30 \%$ had neck dissection for persistent disease) and TORS (12 series with 772 patients, $26 \%$ had adjuvant RT and $41 \%$ had adjuvant chemoradiation) in early T-stage oropharyngeal SCC and found no difference in the overall survival rates at 2 years between the two treatment modalities [15]. Even though the toxicity seems to be lower in TORS-based studies, bias in the selection of patients for TORS, inherently linked to a nonrandomized comparison, precludes any firm conclusion. The results of ongoing randomized studies comparing TORS and IMRT, with sufficiently long follow-up times to reveal the majority of potential toxicities, are awaited. Patients suitable for up-front surgery are those with $T_{1}-T_{2}$ $\mathrm{N}_{0}-\mathrm{N}_{1}$ SCCs who may not require postoperative RT. Thus, definitive RT appears to be an equally good option as primary surgery for all other patients with oropharyngeal SCC.

\section{Supraglottis}

Patients with supraglottic SCC may be stratified into relatively low-volume primary cancers suitable for a supraglottic laryngectomy or definitive RT and advanced SCCs that are best treated with a total laryngectomy and neck dissection usually followed by postoperative (chemo)RT [16, 17]. Patients who are suitable for larynx preservation have small primary tumors $\left(6 \mathrm{~cm}^{3}\right.$ or less as assessed on a contrast-enhanced CT), have a good airway and non-compromised swallowing. Patients with invasion through the cartilage (T4) have been excluded from organ preservation studies following a poor outcome in the VA laryngeal preservation study, which delivered RT alone after a complete or partial response to induction chemotherapy. However, there is recent evidence that delivering concurrent chemo-RT may achieve reasonable local control in some of these patients $[18,19]$.

Patients who are good candidates for a partial laryngectomy must also have adequate pulmonary reserve, so that they can withstand some aspiration postoperatively. In general, patients also tend to have $\mathrm{N}_{0}-\mathrm{N}_{1}$ neck disease, so that postoperative RT can be avoided. It is unwise to operate when positive margins at the primary site are anticipated because high-dose postoperative RT to the primary site after a partial laryngectomy may result in a poor functional outcome. Patients who have low-volume, favorable primary SCCs unsuitable for a partial laryngectomy are treated with definitive RT. After consistent consideration of the above-listed pro et contra argument, the 5 -year locoregional control rates in the range well above $80 \%$ can be expected for early-stage I-II tumors and $\geq 60 \%$ for stage III-IVA tumors with acceptably low $(<5 \%)$ probability of severe late complications, comparing favorably with the surgical series $[16,20]$.

\section{Glottis}

\section{$T 1-T 2$}

T1-T2 glottic SCC may either be treated with definitive RT or transoral resection (TLR), offering the advantage of voice preservation and similar survival rates [21]. Selection of the patients for either of the two treatment modalities should be based on a more comprehensive assessment of particular clinical scenario and not just on the T-stage criterion, e.g., location and extent of the tumor, medical condition of the patient, and expertise of the attending physician. Patients suitable for TLR usually have less advanced and well-defined SCCs confined to the mid-third of one vocal cord; TLR can be done on outpatient basis and repeated several times. Depending on the surgeon, more extensive lesions may be suitable for TLR with a good functional outcome. On the contrary, RT can be used in all patients with early-stage glottis SCC, also with anterior commissure involvement, but is delivered over several weeks, associated with higher cost and cannot be repeated. Open partial laryngectomy is usually reserved for larger lesions or as a salvage procedure for a local recurrence after RT [22]. Patients are irradiated to the larynx only; the risk of subclinical regional disease is low and the neck is not electively irradiated or dissected. Only one prospective study was reported in the literature, randomizing 56 patients with T1aN0 vocal cord SCC over a 10 year recruitment period between the RT arm and TLR arm. No significant difference in the overall voice quality and local control was observed at 24 months post-therapy between the two groups, with a more breathy voice, wider glottis gap, and 
more hoarseness-related inconvenience in daily living reported after TLR [23]. The results of retrospective analyses of large patients' series showed that after RT the initial local control rates for $\mathrm{T} 1$ tumors and $\mathrm{T} 2$ tumors with normal vocal cord mobility were well above $80 \%$ (up to $100 \%$ ) and fell to $76 \%$ at best in the subgroup of $\mathrm{T} 2$ tumors with impaired vocal cord mobility. For these patients, more intensive treatment scenarios should be considered when conservation surgery is not possible, i.e., concomitant platin-based chemoradiation, RT plus cetuximab or altered-fractionated RT over conventional RT alone [24].

\section{T3-T4}

In more advanced laryngeal tumors, selection of patients for organ-sparing non-surgical treatment strategies is essential to assure adequate local control and improved quality of life [i.e., comparable to total laryngectomy and adjuvant radio(chemo)therapy as a gold standard] [25]. In several randomized trials aiming at preserving the larynx, it was spared in approximately two-thirds of the patients with radiation-sensitive tumors who underwent non-surgical treatment. To maximize the chances for local control and a functional organ that allows reasonably good speech and swallowing function, only patients with favorable lowvolume $T_{3}-T_{4}$ SCCs are suitable for definitive RT, i.e., those with primary cancers $4 \mathrm{~cm}^{3}$ or less, good baseline laryngeal function (i.e., airway and swallowing function), and are less than 70 years old to ensure safe administration of systemic drugs and radiotherapy and a satisfactory posttreatment restoration of laryngeal function [26]. However, the optimal combination of RT and systemic therapy is to be defined: in the RTOG 91-11 phase III randomized trial; superior locoregional control and larynx preservation rates were associated with the combination of RT and concurrent cisplatin (over the RT alone arm and the induction chemotherapy followed by RT arm), although the 10-year overall survival was the worst in this arm ( 28 vs. 32 vs. $39 \%$ ) [27]. The reason was fatalities unrelated to laryngeal cancer, most likely unreported toxicity of concurrent chemoradiation, compromising swallowing function and causing silent aspiration. On the other hand, induction chemotherapy can be used to select patients with more/less chemo- and radiosensitive tumors which can guide subsequent treatment decisions $[25,26]$.

\section{Pyriform sinus}

Patients with SCC of the pyriform sinus may be stratified into low-volume favorable primary cancers suitable for larynx preservation either with definitive RT or a partial laryngopharyngectomy (PLP) or more advanced SCCs that are optimally treated with a total laryngectomy combined with a partial or total laryngopharyngectomy, neck dissection, and postoperative RT. Favorable lesions are less than $6-7 \mathrm{~cm}^{3}$ on pretreatment $\mathrm{CT}$, have mobile vocal cords, a good airway, and preserved swallowing function. For T1-T2 pyriform sinus cancers, the outcomes after definitive RT are usually as good as those after PLP with 5 -year local control rates in the range of $85 \%$, so that RT is often the treatment of choice [28-31]. Also, in more advanced but resectable stage III-IV hypopharyngeal SCC, local control after organ preserving combination of RT and chemotherapy seems to favorably compare with the results of primary surgery followed by adjuvant RT [32].

\section{Pharyngeal wall}

$\mathrm{T}_{1}$ and early $\mathrm{T}_{2}$ pharyngeal wall SCCs may be treated with transoral excision. However, these lesions tend to spread beyond their apparent borders in the submucosal lymphatics, so that it may be difficult to obtain adequate margins. Another alternative is to treat patients with pharyngeal wall SCCs with definitive RT. The latter approach is favored at the University of Florida. Mendenhall et al. reported on 170 patients treated with definitive RT at the University of Florida between 1964 and 2009; all living patients had a minimum follow-up of 1.7 years [33]. Thirty patients (18\%) received adjuvant chemotherapy and 43 patients $(25 \%)$ underwent a planned neck dissection. The 5-year LC rates were: $\mathrm{T}_{1}, 93 \% ; \mathrm{T}_{2}, 84 \% ; \mathrm{T}_{3}, 60 \%$; and $\mathrm{T}_{4}, 44 \%$. The 5-year LRC rates were: I, $88 \%$; II, $85 \%$; III, $58 \%$; and IV, $44 \%$. Twenty-five patients (15\%) had severe late complications including permanent feeding tube (17 patients), mandibular osteoradionecrosis necessitating surgery (1 patient), permanent tracheostomy (1 patient), and persistent soft tissue necrosis (6 patients). Four of 6 patients with soft tissue necrosis died as a result of the complication.

\section{Conclusion}

Definitive RT is a good treatment option and often the preferred option for patients with a variety of mucosal head and neck SCCs. The majority of patients present with stage III-IV disease and are likely to benefit from the addition of concomitant chemotherapy. There remain a number of questions including improved target definition as the role of more conformal RT techniques including IMRT and proton beam RT expand, the optimal fractionation schedule particularly when combined with concomitant chemotherapy, de-intensification for HPV-positive non-smokers with oropharyngeal cancers, and the potential role of induction chemotherapy. 
Conflict of interest The authors have no conflicts of interest or financial ties to disclose.

\section{References}

1. Pignon JP, le Maître A, Maillard E, Bourhis J (2009) Metaanalysis of chemotherapy in head and neck cancer (MACH-NC): an update on 93 randomised trials and 17,346 patients. Radiother Oncol 92:4-14

2. Hamoir M, Ferlito A, Schmitz S et al (2012) The role of neck dissection in the setting of chemoradiation therapy for head and neck squamous cell carcinoma with advanced neck disease. Oral Oncol 48:203-210

3. Lee AW, Ng WT, Chan LL et al (2014) Evolution of treatment for nasopharyngeal cancer-success and setback in the intensitymodulated radiotherapy era. Radiother Oncol 110:377-384

4. Mendenhall WM, Morris CG, Amdur RJ, Hinerman RW, Werning JW, Villaret DB (2006) Definitive radiotherapy for squamous cell carcinoma of the base of tongue. Am J Clin Oncol 29:32-39

5. Mendenhall WM, Morris CG, Amdur RJ et al (2006) Definitive radiotherapy for tonsillar squamous cell carcinoma. Am J Clin Oncol 29:290-297

6. Chera BS, Amdur RJ, Hinerman RW et al (2008) Definitive radiation therapy for squamous cell carcinoma of the soft palate. Head Neck 30:1114-1119

7. Mendenhall WM, Amdur RJ, Morris CG, Kirwan JM, Li JG (2010) Intensity-modulated radiotherapy for oropharyngeal squamous cell carcinoma. Laryngoscope 120:2218-2222

8. Graff P, Lapeyre M, Desandes E et al (2007) Impact of intensitymodulated radiotherapy on health-related quality of life for head and neck cancer patients: matched-pair comparison with conventional radiotherapy. Int $\mathbf{J}$ Radiat Oncol Biol Phys 67:1309-1317

9. Feng FY, Kim HM, Lyden TH et al (2010) Intensity-modulated chemoradiotherapy aiming to reduce dysphagia in patients with oropharyngeal cancer: clinical and functional results. J Clin Oncol 28:2732-2738

10. O'Sullivan B, Huang SH, Siu LL et al (2013) Deintensification candidate subgroups in human papillomavirus-related oropharyngeal cancer according to minimal risk of distant metastasis. J Clin Oncol 31:543-550

11. Machtay M, Rosenthal DI, Hershock D et al (2002) Organ preservation therapy using induction plus concurrent chemoradiation for advanced resectable oropharyngeal carcinoma: a University of Pennsylvania Phase II Trial. J Clin Oncol 20:3964-3971

12. Machtay M, Moughan J, Trotti A et al (2008) Factors associated with severe late toxicity after concurrent chemoradiation for locally advanced head and neck cancer: an RTOG analysis. J Clin Oncol 26:3582-3589

13. Denittis AS, Machtay M, Rosenthal DI et al (2001) Advanced oropharyngeal carcinoma treated with surgery and radiotherapy: oncologic outcome and functional assessment. Am J Otolaryngol 22:329-335

14. Haughey BH, Hinni ML, Salassa JR et al (2011) Transoral laser microsurgery as primary treatment for advanced-stage oropharyngeal cancer: a United States multicenter study. Head Neck 33:1683-1694

15. de Almeida JR, Byrd JK, Wu R et al (2014) A systematic review of transoral robotic surgery and radiotherapy for early oropharynx cancer: a systematic review. Laryngoscope 124:2096-2102

16. Hinerman RW, Mendenhall WM, Amdur RJ, Stringer SP, Villaret DB, Robbins KT (2002) Carcinoma of the supraglottic larynx: treatment results with radiotherapy alone or with planned neck dissection. Head Neck 24:456-467

17. Mendenhall WM, Morris CG, Amdur RJ, Hinerman RW, Mancuso AA (2003) Parameters that predict local control after definitive radiotherapy for squamous cell carcinoma of the head and neck. Head Neck 25:535-542

18. Worden FP, Moyer J, Lee JS et al (2009) Chemoselection as a strategy for organ preservation in patients with T4 laryngeal squamous cell carcinoma with cartilage invasion. Laryngoscope 119:1510-1517

19. Mouw KW, Solanki AA, Stenson KM et al (2012) Performance and quality of life outcomes for T4 laryngeal cancer patients treated with induction chemotherapy followed by chemoradiotherapy. Oral Oncol 48:1025-1030

20. Buglione M, Pedretti S, Costa L et al (2015) Clinical outcomes and toxicity after exclusive versus postoperative radiotherapy in supraglottic cancer: new solutions for old problems? The case of stage I and II disease. Radiol Med [Epub ahead of print]

21. Mendenhall WM, Takes RP, Shah JP et al (2014) Current treatment of T1N0 squamous cell carcinoma of the glottic larynx. Eur Arch Otorhinolaryngol [Epub ahead of print]

22. Mendenhall WM, Werning JW, Hinerman RW, Amdur RJ, Villaret DB (2004) Management of T1-T2 glottic carcinomas. Cancer 100:1786-1792

23. Aaltonen LM, Rautiainen N, Sellman J et al (2014) Voice quality after treatment of early vocal cord cancer: a randomized trial comparing laser surgery with radiation therapy. Int $\mathrm{J}$ Radiat Oncol Biol Phys 90:255-260

24. Hartl DM, Ferlito A, Brasnu DF et al (2011) Evidence-based review of treatment options for patients with glottic cancer. Head Neck 33:1638-1648

25. Strojan P, Haigentz M Jr, Bradford CR et al (2013) Chemoradiotherapy vs. total laryngectomy for primary treatment of advanced laryngeal squamous cell carcinoma. Oral Oncol 49:283-286

26. Lefebvre JL, Ang KK (2009) Larynx preservation clinical trial design: key issues and recommendations-a consensus panel summary. Head Neck 31:429-441

27. Forastiere AA, Zhang Q, Weber RS et al (2013) Long-term results of RTOG 91-11: a comparison of three nonsurgical treatment strategies to preserve the larynx in patients with locally advanced larynx cancer. J Clin Oncol 31:845-852

28. Rabbani A, Amdur RJ, Mancuso AA et al (2008) Definitive radiotherapy for T1-T2 squamous cell carcinoma of pyriform sinus. Int J Radiat Oncol Biol Phys 72:351-355

29. Nakamura K, Shioyama Y, Kawashima M et al (2006) Multiinstitutional analysis of early squamous cell carcinoma of the hypopharynx treated with radical radiotherapy. Int J Radiat Oncol Biol Phys 65:1045-1050

30. Nakajima A, Nishiyama K, Morimoto M et al (2012) Definitive radiotherapy for T1-2 hypopharyngeal cancer: a single-institution experience. Int J Radiat Oncol Biol Phys 82:e129-e135

31. Blanchard P, Tao Y, Veresezan O et al (2012) Definitive radiotherapy for squamous cell carcinoma of the pyriform sinus. Radiother Oncol 105:232-237

32. Kim JW, Kim MS, Kim SH et al (2015) Definitive chemoradiotherapy versus surgery followed by adjuvant radiotherapy in resectable stage III/IV hypopharyngeal cancer. Cancer Res Treat [Epub ahead of print]

33. Mendenhall WM, Morris CG, Kirwan JM, Amdur RJ, Vaysberg M, Werning JW (2012) Definitive radiation therapy for squamous cell carcinoma of the pharyngeal wall. Pract Radiat Oncol 2:e113-e119 